\title{
Dental Health Status in Hospitalized Psychiatric Patients in Sistan and Baluchestan Province, Iran
}

\author{
Leila Farhadmollashahi ${ }^{1,} ;$ Kobra Lashkaripour $^{2}$; Nour-Mohammad Bakhshani ${ }^{2}$; Maryam \\ Faghihinia ${ }^{1}$
}

${ }^{1}$ Department of Oral Medicine, School of Dentistry, Zahedan University of Medical Sciences, Zahedan, IR Iran

${ }^{2}$ Department of Psychiatry, School of Medicine, Zahedan University of Medical Sciences, Zahedan, IR Iran

${ }^{*}$ Corresponding author: Leila Farhadmollashahi, Department of Oral Medicine, School of Dentistry, Zahedan University of Medical Sciences, Zahedan, IR Iran. Tel: +98-9151430868, Fax:+98-5412414003, E-mail: Im_farhad@yahoo.com

Received: June 30, 2014; Revised: October 29, 2014; Accepted: October 31, 2014

\begin{abstract}
Background: Psychiatric disorders to various reasons such side effects of psychiatric drugs, poor oral hygiene and poor diet and are known as risk factors for development oral health problems.

Objectives: This study was conducted to determine the dental status in hospitalized psychiatric patients in Sistan and Baluchestan province, Iran.

Patients and Methods: This cross-sectional study was conducted on 120 patients in the only psychiatric hospital in this province. Dental status was assessed through measuring the mean of the number of decayed, missing and filled teeth (DMFT index). The collected data was analyzed using t-test, analysis of variance and Tukey test.

Results: The mean DMFT score among the patients was $13.75 \pm 7.8$. There were statistically significant associations between age, duration of mental illness, duration of medication usage, education level, and tooth brushing frequency and DMFT index $(\mathrm{P}<0.05)$. Differences of DMFT Indexes with sex and type of psychiatric disorders were not statistically significant.

Conclusions: The results of this study indicated that effective dental care is needed for improvement of the dental health status of hospitalized patients with psychiatric disorders.
\end{abstract}

Keywords:Psychotic Disorder; Dental Health Services; DMF Indices

\section{Background}

Mental illnesses are among the most common debilitating diseases (1) and are considered as the risk factors of oral health problems (2). Today, more than 450 million people in the world have mental illnesses $(1,2)$. They engage people at all economic, social, and national levels $(2,3)$. The studies carried out in Iran show that the prevalence of such disorders varies between $10.8 \%$ and $21 \%$ (1). Individuals with severe mental illnesses have a higher risk of oral illness as compared with healthy people, which is due to different factors such as type and severity of the illness, poor oral health, specific dental phobias, problem accessing healthcare centers, inappropriate diet, lack of personal understanding of oral care problems, and sideeffects of psychiatric medications (2). The psychologicalsocial impacts of oral health problems affect the quality of life considerably; this affects eating, speaking, and social and psychological aspects of life (2). Dental caries and periodontal diseases are the most common diseases engaging the oral health $(2,4)$. Consumption of psychiatric medications reduces salivary flow rate and its buffering capacity and causes dental caries in the presence of a normal diet (5). Dental treatment of these patients will be difficult due to lack of motivation, detachment, limited partnership, mobility problems, fear of treatment, poor adaptation to new prostheses, and financial considerations (2). In addition, nursing staff of hospitals lack necessary knowledge, ability and incentive to provide conditions for oral heath cares of these patients (5). Several studies were conducted on oral health care of patients with psychiatric disorders in Denmark (6), Italy (7), Spain (8), India (9), and Turkey (10) and proved that the mental illnesses and the medications consumed for these diseases may increase the severity of dental illnesses, especially caries.

\section{Objectives}

Among the unique communities, patients with psychiatric disorders need special cares. Few studies have been conducted for determining the oral health condition of the hospitalized patients with mental disorders in Iran. No study has been carried out in this concern in Sistan and Baluchestan province, the vastest province in southeastern Iran. With respect to cultural and social diversity, life style of different societies, and necessity of attention to the health status of the individuals at risk, the present study was conducted in the only psychiatric-specialized hospital

Copyright (C2014, Health Promotion Research Center. This is an open-access article distributed under the terms of the Creative Commons Attribution-NonCommercial 4.0 International License (http://creativecommons.org/licenses/by-nc/4.0/) which permits copy and redistribute the material just in noncommercial usages, provided the original work is properly cited. 
in the province, aiming at studying the oral health of the hospitalized patients with mental disorders.

\section{Patients and Methods}

This descriptive-analytical study was performed in the only psychiatric-specialized hospital in Sistan and Baluchestan province in 2010. As the minimum hospitalization duration of patients in the psychiatric hospital was four weeks, diagnostic evaluations were carried out based on DSM-IV-TR (diagnostic and statistical manual of mental disorders) criteria on all the patients during weekly visits by a psychologist (11). The patients' levels of cooperation were studied, the study purpose was completely explained to the patients, and the individuals were included in the study voluntarily after obtaining written consents. The examinations of teeth were carried out by a senior dental student. Dental caries condition was examined based on the World Health Organization (WHO) criteria (12) using a mirror, disposable catheters, and a flashlight. The number of decayed, missing and filled teeth (DMFT index) was used for examining dental caries. This index consists of three components: 1 ) decayed tooth (component D) as a tooth with active caries, 2) missing tooth (component $\mathrm{M}$ ) as a lost tooth due to caries or other reasons, and 3) filled tooth (component F) as a restored tooth due to caries. DMFT was considered as sum of three components $\mathrm{D}+\mathrm{M}+\mathrm{F}$ (5). Other data including demographic specifications, type of consumed drug, treatment duration and mental illness duration were obtained by referring to the medical files and interviewing the patients. Data were analyzed through the tables and indices of descriptive-analytic statistics, ANOVA, Tukey's test and t-test, using SPSS-17.

\section{Results}

This study examined 120 patients with mental disor- ders (28.3\% females and 71.7\% males), 15-64 years old, with a mean age of $34.5 \pm 11.41$. The mean of patients' DMFT index was obtained as $13.75 \pm 7.8$. Table 1 shows the means of DMFT index in terms of the demographic specifications. The results of ANOVA showed a significant difference in the mean of DMFT index in terms of the age groups $(\mathrm{P}<0.001)$. Comparing the mean of DMFT index based on Tukey's test showed that the differences between adolescent and middle-aged groups $(\mathrm{P}<0.001)$ and between adult and middleaged groups $(\mathrm{P}<0.001)$ were significant.

Comparing the mean of DMFT index and D, M, F quantities in terms of gender using t-test showed no significant difference between the mean of quantities and the mean of DMFT index of the patients $(P=0.29)$. The results of one-way ANOVA showed a significant difference in the mean of DMFT index in terms of level of education ( $\mathrm{P}=$ 0.03). Comparing the mean of DMFT index of the patients in terms of level of education using Tukey's test showed that the difference between the illiterate and pre-diploma groups was significant $(\mathrm{P}=0.02)$. The results of oneway ANOVA showed a significant difference in the mean of DMFT index in terms of number tooth brushing ( $\mathrm{P}<$ 0.001). Comparing the mean of DMFT index of the patients using Tukey's test in terms of the brushing method showed a significant difference between the group that did not brush their teeth and the one that sometimes brush $(\mathrm{P}<0.001)$ and between the group that did not brush and the one that brush everyday $(\mathrm{P}=0.03)$.

Table 2 shows the mean of DMFT index in terms of illness specifications. Here, the number of those with schizophrenia exceeded the ones with other types of mental diseases. Tukey's test results showed no significant difference between the mean of quantities and DMFT index in terms of type of illness ( $P>0.05)$, whereas one-way ANOVA showed a significant difference among DMFT index, illness duration, and drug consumption $(\mathrm{P}<0.05)$.

\begin{tabular}{|c|c|c|c|c|c|}
\hline Variables & DMFT & Missing & Filled & Decayed & Results \\
\hline \multicolumn{6}{|l|}{ Age Group, y } \\
\hline Adolescents & $8.60 \pm 2.70$ & $2 \pm 2$ & $0 \pm 0.0$ & $6.6 \pm 3.36$ & $5(4.16)$ \\
\hline Youth & $10.98 \pm 6.16$ & $4.26 \pm 5.25$ & $0.3 \pm 1.71$ & $6.5 \pm 3.76$ & $72(60)$ \\
\hline Adults & $19 \pm 8.12$ & $13.51 \pm 8.93$ & $0 \pm 0.0$ & $5.5 \pm 3.67$ & $43(35.84)$ \\
\hline \multicolumn{6}{|l|}{ Gender } \\
\hline Female & $15.70 \pm 8.54$ & $9.58 \pm 8.69$ & $0.08 \pm 0.51$ & $6.05 \pm 3.96$ & $34(28.30)$ \\
\hline Male & $12.98 \pm 7.49$ & $6.65 \pm 7.73$ & $0.22 \pm 1.54$ & $6.16 \pm 3.63$ & $86(71.70)$ \\
\hline \multicolumn{6}{|l|}{ Education } \\
\hline Illiterate & $16.02 \pm 7.2$ & $9.57 \pm 7.25$ & $0 \pm 0.0$ & $6.45 \pm 3.60$ & $42(35)$ \\
\hline Pre-diploma & $11.86 \pm 7.21$ & $5.75 \pm 7$ & $0.08 \pm 0.43$ & $6.13 \pm 3.80$ & $52(43.30)$ \\
\hline Post-diploma & $13.88 \pm 9.05$ & $7.57 \pm 10.57$ & $0.69 \pm 2.78$ & $5.61 \pm 3.67$ & $26(21.70)$ \\
\hline \multicolumn{6}{|c|}{ Tooth brushing habits } \\
\hline Never & $16.75 \pm 8.53$ & $10.21 \pm 9.56$ & $0.60 \pm 0.41$ & $6.50 \pm 3.88$ & $52(43.30)$ \\
\hline Sometimes & $11.31 \pm 6.25$ & $5.36 \pm 5.82$ & $0.02 \pm 0.14$ & $5.39 \pm 3.37$ & $47(39.20)$ \\
\hline Everyday & $11.81 \pm 7.15$ & $5.47 \pm 6.64$ & $0.9 \pm 3.08$ & $5.66 \pm 4.11$ & $21(17.50)$ \\
\hline
\end{tabular}

\footnotetext{
a Data are presented as Mean \pm SD or No. (\%).
} 
Farhadmollashahi L et al.

\begin{tabular}{lccccc}
\hline \multicolumn{1}{l}{ Table 2. DMFT Index, Missing, Filled, and Decayed Quantities in Terms of Illness Characteristics ${ }^{\mathrm{a}}$} & & \\
\hline \multicolumn{1}{l}{ Variables } & DMFT & Missing & Filled & Decayed & Results \\
\hline $\begin{array}{l}\text { Type of illness } \\
\text { Schizophrenia }\end{array}$ & $15.22 \pm 6.62$ & $8.31 \pm 6.89$ & $0.06 \pm 0.45$ & $6.86 \pm 3.60$ & $44(36.70)$ \\
\hline Bipolar disorders & $13.48 \pm 7.86$ & $7.11 \pm 7.04$ & $0.11 \pm 0.57$ & $6.4 \pm 3.74$ & $27(22.50)$ \\
\hline Major depression & $15.81 \pm 8.86$ & $11 \pm 10.95$ & $0.00 \pm 0.00$ & $4.81 \pm 2.36$ & $11(9.20)$ \\
\hline Other mental illnesses & $11.57 \pm 8.85$ & $6.04 \pm 9.05$ & $0.66 \pm 3.05$ & $4.85 \pm 3.85$ & $21(17.50)$ \\
\hline Addiction & $11.76 \pm 8.78$ & $5.41 \pm 9.11$ & $0.11 \pm 0.33$ & $6.23 \pm 4.16$ & $17(14.20)$ \\
\hline Illness duration, $\mathbf{y}$ & & & & & \\
\hline$<2$ & $9.66 \pm 8.20$ & $4.78 \pm 8.39$ & $0.45 \pm 2.43$ & $4.54 \pm 3.51$ & $33(27.50)$ \\
\hline $2-5$ & $13.92 \pm 6.87$ & $6.90 \pm 7.23$ & $0.16 \pm 0.65$ & $6.88 \pm 3.26$ & $41(34.20)$ \\
\hline$>5$ & $16.60 \pm 7.31$ & $10 \pm 8.09$ & $0.00 \pm 0.00$ & $6.60 \pm 3.97$ & $46(38.30)$ \\
\hline Treatment duration, mo & & & & & \\
\hline$<6$ & $10.30 \pm 8.12$ & $4.80 \pm 8.03$ & $0.40 \pm 2.21$ & $5.20 \pm 3.98$ & $40(43.30)$ \\
\hline $6-12$ & $13 \pm 3.60$ & $5.33 \pm 5.85$ & $0.00 \pm 0.00$ & $8 \pm 2.64$ & $3(2.50)$ \\
\hline$>12$ & $15.58 \pm 7.27$ & $8.96 \pm 7.89$ & $0.07 \pm 0.48$ & $6.54 \pm 3.54$ & $77(64.20)$ \\
\hline
\end{tabular}

${ }^{\mathrm{a}}$ Data are presented as Mean \pm SD or No. (\%).

A significant difference was observed while comparing the mean of DMFT quantity and Tukey's test based on the years having the illness, between illness duration more than five years and less than two years $(\mathrm{P}<0.001)$ and illness duration between two and five years with the duration shorter than two years $(\mathrm{P}=0.04)$. The results of the mean of DMFT index of the patients in terms of drug consumption duration using Tukey's test showed a significant difference between the DMFT quantity in patients with drug consumption history shorter than six months and longer than one year $(\mathrm{P}<0.001)$.

\section{Discussion}

In our study, the mean of DMFT index was $13.75 \pm 7.8$, which was higher than the DMFT of the normal population under study in the province $(10.05 \pm 7.18)(13)$. Our findings were fully compatible with the ones of Chu et al. (14). Findings of Jovanovic et al. (15), Zusman et al. (16), Velasco-Ortega et al. (5), Ghaffarinejad et al. (17), and Nikfarjam et al. (18) were far beyond the findings of our study. A similar study in Kerman and Chaharmahal and Bakhtiari provinces reported the means of DMFT index in patients with mental disorders as 19.74 and 19.43, respectively $(17,18)$. Conversely, studies of Kebede et al. (2), Kumar et al. (3), Adeniyi et al. (19), and Rekha et al. (20) reported the DMFT indexes lower than those of our study. Due to the difference between the age groups under study, type of consumed drugs, mental illness durations, and accessible treatment levels, it was difficult to compare the results of the present study with other studies, which was considered as a limitation of the study. Another limitation of our study was lack of a control group, which was resolved with respect to conducting a study at the same time on the normal population living in the province to study the DMFT mean.
The mean number of decayed teeth in our study was 6.1, which was compatible with the study by Ramon et al. in which it was 6.2 (21), whereas Kebede et al. (2), Kumar et al. (3) Velasco-Ortega et al. (5), and Lewis et al. (22) reported higher values. Here, the mean of missing teeth was 7.4, which was lower than the values reported by Kumar et al. (3) and Velasco-Ortega et al. (5) and higher than the ones reported by Kebede et al. (2). In this study, the mean of the number of filled teeth was compatible with those of Kebede et al. (2) and Adeniyi et al. (19) reports and lower than the findings reported by Lewis et al. (22) and Zusman et al. (16). This may bring up inadequate access of the patients under the study to dentistry services. In our study, the mean of DMFT index increased with increasing age, which was compatible with the findings of the earlier studies $(2,15)$. There were no statistically significant differences among the mean of D, M, F quantities, DMFT index, and gender of the patients under study, whereas a significant difference existed between gender and DMFT index in the study of Kebede et al. (2) and the male gender was introduced as a risk factor of dental caries. In our study, the mean of DMFT index increased with mental illness duration. Kumar et al. (3) and Jovanovic et al. (15) also reported a relationship between the index and illness duration. This can be justified by the fact that individuals with severe mental diseases may be reluctant to dental treatments due to fear of pain, dental phobia or high dental costs (4). Here, the mean of DMFT index increased in terms of duration of drug consumption. Consumption of psychotropic drugs may reduce the salivary flow rate and cause xerostomia $(2,4)$. Xerostomia may reduce the quality of life, increase formation of plaque and calculus, and increase the incidence of caries, gingivitis, and periodontitis (4).

The mean of DMFT index in our study was higher than 
that of the normal population under study in this province. Such findings indicate attention to the oral health problems of this special group of patients and confirm the necessity of cooperation of dentists in the treatment system of patients with mental disorders.

\section{Acknowledgements}

The authors would like to thank the Research Council of Zahedan Dentistry Faculty and the Research Ethics Committee and nursing staff of Baharan Psychiatric Hospital who assisted with conducting this research.

\section{Authors' Contributions}

Study concept: Leila Farhadmollashahi; statistical analysis: Nour-Mohammad Bakhshani; study supervision: Nour-Mohammad Bakhshani and Kobra Lashkari Pour; data collection: Maryam Faghihi; writing of the manuscript: Leila Farhadmollashahi.

\section{References}

1. Farhoudian A, Sharifi V, Amini H, Basirnia A, Mesgarpour B, Mansouri N, et al. Prevalence of psychiatric disorders in Iran: A systematic review. Iran J Psychiatry. 2007;2(4):137-50.

2. Kebede B, Kemal T, Abera S. Oral health status of patients with mental disorders in southwest Ethiopia. PLoS One. 2012;7(6).

3. Kumar M, Chandu GN, Shafiulla MD. Oral health status and treatment needs in institutionalized psychiatric patients: one year descriptive cross sectional study. Indian J Dent Res. 2006; 17(4):171-7.

4. Kisely S, Quek LH, Pais J, Lalloo R, Johnson NW, Lawrence D. Advanced dental disease in people with severe mental illness: systematic review and meta-analysis. Br J Psychiatry. 2011; 199(3):187-93.

5. Velasco-Ortega E, Segura-Egea JJ, Cordoba-Arenas S, JimenezGuerra A, Monsalve-Guil L, Lopez-Lopez J. A comparison of the dental status and treatment needs of older adults with and without chronic mental illness in Sevilla, Spain. Med Oral Patol Oral Cir Bucal. 2013;18(1):e71-5.

6. Hede B, Petersen PE. Self-assessment of dental health among Danish noninstitutionalized psychiatric patients. Spec Care Dentist. 1992;12(1):33-6.

7. Angelillo IF, Nobile CG, Pavia M, De Fazio P, Puca M, Amati A. Dental health and treatment needs in institutionalized psychiatric patients in Italy. Community Dent Oral Epidemiol.1995;23(6):360-4.
8. Velasco E, Bullon P. Periodontal status and treatment needs among Spanish hospitalized psychiatric patients. Spec Care Dentist.1999;19(6):254-8.

9. Kenkre AM, Spadigam AE. Oral health and treatment needs in institutionalized psychiatric patients in India. Indian J Dent Res. 2000;11(1):5-11.

10. Gurbuz O, Alatas G, Kurt E, Issever H, Dogan F. Oral health and treatment needs of institutionalized chronic psychiatric patients in Istanbul, Turkey. Community Dent Health. 2010;27(3):151-7.

11. Kawa S, Giordano J. A brief historicity of the Diagnostic and Statistical Manual of Mental Disorders: issues and implications for the future of psychiatric canon and practice. Philos Ethics Humanit Med. 2012;7:2.

12. Ishii T, Yoshida S. [Oral health surveys--basic methods--fundamental and practical problems of oral health surveys by WHO]. Shikai Tenbo. 1978;51(4):762-72.

13. Rigi Ladiz M, Ghanbariha M, Badiee M, Abdolazimi Z. The Relationship between Dental Caries and Some socio - economic Factors in 35-44 years old Adults in some urban Areas of sistan \& Baluchestan.J Shaheed Sadoughi Univ Med Sci. 2012;20(4):454-63.

14. Chu KY, Yang NP, Chou P, Chiu HJ, Chi LY. Factors associated with dental caries among institutionalized residents with schizophrenia in Taiwan: a cross-sectional study. BMC Public Health. 2010;10:482.

15. Jovanovic S, Milovanovic SD, Gajic I, Mandic J, Latas M, Jankovic L Oral health status of psychiatric in-patients in Serbia and implications for their dental care. Croat Med J. 2010;51(5):443-50.

16. Zusman SP, Ponizovsky AM, Dekel D, Masarwa AE, Ramon T, Natapov L, et al. An assessment of the dental health of chronic institutionalized patients with psychiatric disease in Israel. Spec Care Dentist. 2010;30(1):18-22.

17. Ghaffarinejad A, Chamani G, Zarei MR, Mehdizadeh Zare Anari A Aghaabasi S. Oral and dental health in of hospitalized psychiatric patients: A cross sectional and comparative study in kerman city, iran. Eur Psychiat. 2013;28:1.

18. Nikfarjam M, Foruzandeh N, Parvin N. Dental Health Status of Schizophrenic Patients in the Chronic Psychiatric Care Center in the Province of Chaharmahal va Bakhtiyary. J Rehabil. 2011;12(2):14-9.

19. Adeniyi AA, Ola BA, Edeh CE, Ogunbanjo BO, Adewuya AO. Dental status of patients with mental disorders in a Nigerian teaching hospital: a preliminary survey. Spec Care Dentist. 2011;31(4):134-7.

20. Rekha R, Hiremath SS, Bharath S. Oral health status and treatment requirements of hospitalized psychiatric patients in Bangalore city: a comparative study. J Indian Soc Pedod Prev Dent. 2002;20(2):63-7.

21. Ramon T, Grinshpoon A, Zusman SP, Weizman A. Oral health and treatment needs of institutionalized chronic psychiatric patients in Israel. Eur Psychiatry. 2003;18(3):101-5.

22. Lewis S, Jagger RG, Treasure E. The oral health of psychiatric inpatients in South Wales. Spec Care Dentist. 2001;21(5):182-6. 